\title{
Interaction of the signal transduction protein GlnJ with the cellular targets AmtB1, GlnE and $G \ln D$ in Rhodospirillum rubrum: dependence on manganese, 2-oxoglutarate and the ADP/ATP ratio
}

Correspondence

Stefan Nordlund

stefan@dbb.su.se

Received 13 February 2008

Revised 7 May 2008

Accepted 13 May 2008

\author{
Pedro Filipe Teixeira, Anders Jonsson, Martina Frank, He Wang \\ and Stefan Nordlund
}

Department of Biochemistry and Biophysics, Stockholm University, SE-10691 Stockholm, Sweden

\begin{abstract}
The PII family of signal transduction proteins is widespread amongst the three domains of life, and its members have fundamental roles in the general control of nitrogen metabolism. These proteins exert their regulatory role by direct protein-protein interaction with a multitude of cellular targets. The interactions are dependent on the binding of metabolites such as ATP, ADP and 2oxoglutarate (2-OG), and on whether or not the PII protein is modified. In the photosynthetic nitrogen-fixing bacterium Rhodospirillum rubrum three PIl paralogues have been identified and termed GlnB, GlnJ and GlnK. In this report we analysed the interaction of GlnJ with known cellular targets such as the ammonium transporter AmtB1, the adenylyltransferase GlnE and the uridylyltransferase GlnD. Our results show that the interaction of $\mathrm{Gln} J$ with cellular targets is regulated in vitro by the concentrations of manganese and 2-OG and the ADP: ATP ratio. Furthermore, we show here for the first time, to our knowledge, that in the interactions of GlnJ with the three different partners, the energy signal (ADP : ATP ratio) in fact overrides the carbon/ nitrogen signal (2-OG). In addition, by generating specific amino acid substitutions in GlnJ we show that the interactions with different cellular targets are differentially affected, and the possible implications of these results are discussed. Our results are important to further the understanding of the regulatory role of PII proteins in $R$. rubrum, a photosynthetic bacterium in which the nitrogen fixation process and its intricate control mechanisms make the regulation of nitrogen metabolism even more complex than in other studied bacteria.
\end{abstract}

\section{INTRODUCTION}

Nitrogen is an essential component of most biological macromolecules and although abundant in the atmosphere, this form is chemically unavailable to most organisms. Only a few prokaryotic organisms (the diazotrophs) have the ability to use molecular dinitrogen to produce ammonium ions/ammonia (hereafter 'ammonium' is used to designate both forms). The photosynthetic bacterium Rhodospirillum rubrum is a metabolically versatile organism that can fix nitrogen in the reaction catalysed by the metalloenzyme complex nitrogenase. The activity of nitrogenase is strictly controlled by reversible post-translational ADP ribosylation in response to socalled 'switch-off' effectors, e.g. addition of ammonium or exposure of the cells to darkness (Nordlund \& Ludden, 2004).

Abbreviations: GS, glutamine synthetase; 2-OG, 2-oxoglutarate.

A supplementary figure showing models of the structure of $R$. rubrum GlnJ is available with the online version of this paper.
However, if available, ammonium can also be transported from the outside medium through a family of membrane proteins, the ammonium transporters (Amt). These proteins are ubiquitous among archaea, bacteria, fungi and plants, and are related to the Rhesus protein family of animals (Khademi et al., 2004).

Ammonium is further assimilated into glutamate/glutamine through the glutamine synthetase (GS)/glutamate synthase pathway (Merrick \& Edwards, 1995). In $R$. rubrum GS activity is strictly regulated by a mechanism that involves the PII signalling proteins and which is sensitive to the concentration of metabolites such as 2oxoglutarate (2-OG) and, indirectly, glutamine (Jonsson et al., 2007).

The PII family of signalling proteins is widespread amongst bacteria, archaea and eukarya (plants), and these proteins are involved in the general control of nitrogen metabolism (Arcondeguy et al., 2001). The most extensively studied members are GlnB and GlnK from Escherichia coli. These 
proteins are soluble, trimeric and known to bind metabolites such as ATP (Xu et al., 1998) and 2-OG (Atkinson \& Ninfa, 1999; Jiang et al., 1998a; Kamberov et al., 1995). The PII monomers fold in a double $\beta \alpha \beta$ motif, forming a compact barrel structure in the trimeric form (Xu et al., 1998). Three loops are observable in each monomer, designated the B-, C- and T-loops. The T-loop has been shown to be especially important for interaction with different cellular targets (Jiang et al., 1997).

In most proteobacteria the PII proteins are also regulated through reversible covalent modification with UMP at a specific tyrosine residue (Tyr51) located in the T-loop (Arcondeguy et al., 2001). This modification is catalysed by the bifunctional enzyme uridylyltransferase/uridylylremovase (UTase/UR) (GlnD; encoded by $g \ln D$ ), which in E. coli 'senses' the cellular nitrogen status through the concentration of glutamine (Jiang et al., 1998b). However, other types of modification can occur, such as adenylylation in Streptomyces coelicolor (Hesketh et al., 2002) and Corynebacterium glutamicum (Strosser et al., 2004), and phosphorylation in cyanobacteria (Forchhammer \& Tandeau de Marsac, 1994). In other organisms, such as Bacillus subtilis (Heinrich et al., 2006) and Arabidopsis thaliana (Smith et al., 2004), no PII protein modification has been demonstrated. In R. rubrum, three PII paralogues have been identified (Zhang et al., 2001), termed GlnB, GlnK and GlnJ, which are subjected to UMP modification at Tyr51 (Jonsson \& Nordlund, 2007). Although the amino acid sequence of the three proteins is very similar, they show both distinct and overlapping functions in the cell, including post-translational regulation of nitrogenase and GS as well as activation of gene transcription (Wang et al., 2005; Zhang et al., 2001, 2005, 2006b). At the molecular level, the effect of PII proteins on the post-translational regulation of GS has been analysed in greater detail in $R$. rubrum (Jonsson et al., 2007). GS is regulated by reversible adenylylation catalysed by the bifunctional enzyme GlnE. It has been shown recently that a PII protein $(\mathrm{Gln} B, \mathrm{Gln}$ J or $\mathrm{GlnK}$ ) is required in vitro to activate the adenylylation activity of GlnE (leading to inactivation of GS) and also that this reaction is inhibited by 2-OG. The reverse reaction, deadenylylation (GS activation) seems to be unregulated in R. rubrum (Jonsson et al., 2007).

The $g \ln B$ gene is co-transcribed with $g \ln A$ (the gene encoding GS) (Johansson \& Nordlund, 1996), while both $g \ln J$ and $g \ln K$ are linked to genes encoding putative ammonium transporters of the Amt family, amtB1 and amtB2, respectively (Zhang et al., 2006b). It has been shown that an $R$. rubrum mutant lacking amtB1 has an impaired nitrogenase 'switch-off response and fails to sequester GlnJ to the membrane (Wang et al., 2005; Zhang et al., 2006b). Also, a similar phenotype has been observed in an Azospirillum brasilense mutant lacking amtB (Huergo et al., 2006).

The existence of a genetic linkage between the bacterial $g \ln K$-like and amt genes (usually in the same operon)
(Thomas et al., 2000) suggests a physical interaction, which has been observed in several prokaryotes, including bacteria (Durand \& Merrick, 2006; Heinrich et al., 2006; Huergo et al., 2007; Wolfe et al., 2007) and archaea (Yildiz et al., 2007). The functional significance of this association has been investigated in $E$. coli and it has been suggested that the primary functions of the AmtB-GlnK complex are the sensing of the extracellular ammonium availability and therefore the regulation of ammonium transport through AmtB (Javelle et al., 2004). In fact, the recently determined 3D structure of the E. coli AmtB-GlnK complex clearly shows that GlnK acts as a 'plug', affecting ammonia conduction through the channel (Conroy et al., 2007).

The formation of a complex between Amt and GlnK is affected by several metabolites, which vary between organisms. In $R$. rubrum, the association between the GlnK homologue GlnJ and the ammonium transporter AmtB1 has been demonstrated in a recent report that describes the formation of a stable complex that is dissociated through synergistic binding of ATP and 2-OG (Wolfe et al., 2007). One of the fundamental questions concerning the Amt-PII complexes is the specificity of the interaction towards the GlnB- or the GlnK-like proteins. The question of the specificity of the Amt-PII interaction in $R$. rubrum was also addressed in the same study, and a preference was shown for GlnJ association to AmtB1 (Wolfe et al., 2007), thus confirming a previous report (Wang et al., 2005).

In the present study we have analysed the interaction of $R$. rubrum GlnJ with membrane-bound AmtB1, and also with the cytosolic targets $\mathrm{GlnE}$ and $\mathrm{Gln} \mathrm{D}$, and have shown that the interactions are sensitive to the concentrations of manganese and 2-OG and the ADP:ATP ratio. Furthermore, we have shown that the dissociation of GlnJ from AmtB1 and subsequent uridylylation by $G \ln D$ are controlled by the same factors, suggesting a functional coupling between the two processes. Also, by constructing several GlnJ variants, we have highlighted the importance of the T-loop residues Gln39, Arg47 and Lys58 in the interactions with the three cellular targets.

\section{METHODS}

Bacterial growth. Bacterial strains and plasmids used in this study are listed in Table 1. Plasmid-harbouring E. coli strains were routinely grown on selective Luria-Bertani medium containing antibiotics at the following final concentrations: $50 \mu \mathrm{g}$ ampicillin (Ap) $\mathrm{ml}^{-1}, 15 \mu \mathrm{g}$ tetracycline $(\mathrm{Tc}) \mathrm{ml}^{-1}$ and $34 \mu \mathrm{g}$ chloramphenicol $(\mathrm{Cm}) \mathrm{ml}^{-1} . R$. rubrum S1 was grown in a medium described elsewhere (Ormerod et al., 1961) containing $40 \mathrm{mM}$ malate, under an atmosphere of $95 \%$ $\mathrm{N}_{2} / 5 \% \mathrm{CO}_{2}$, at $25-30{ }^{\circ} \mathrm{C}$.

Site-directed mutagenesis of $\boldsymbol{g} / \boldsymbol{n} \boldsymbol{J}$. The R47A and K58A GlnJ variants were generated by standard PCR-mediated site-directed mutagenesis using the QuikChange kit (Stratagene). The primers used contained a $2 \mathrm{bp}$ mismatch that converted the codons from arginine or lysine to alanine at positions 47 and 58, respectively. The template used was pETGlnJ (Jonsson et al., 2007), and the new plasmids were 
Table 1. Bacterial strains and plasmids used in this study

\begin{tabular}{|c|c|c|}
\hline Strain or plasmid & Genotype and/or relevant characteristics & Reference or source \\
\hline \multicolumn{3}{|l|}{ Strains } \\
\hline \multicolumn{3}{|l|}{ E. coli } \\
\hline BL21 (DE3) pLysS & Host for overexpression of pET15b derivatives, $\mathrm{Cm}^{\mathrm{r}}$ & Invitrogen \\
\hline BL21 Star (DE3) & Host for overexpression of pETGlnE & Invitrogen \\
\hline RB 9040 & $\Delta g \ln D$; host for overexpression of pGEX-GlnD, $\mathrm{Tc}^{\mathrm{r}}$ & Bueno et al. (1985) \\
\hline \multicolumn{3}{|l|}{ R. rubrum } \\
\hline S1 & Wild-type & \\
\hline \multicolumn{3}{|l|}{ Plasmids } \\
\hline pETGlnE & pET101 derivative containing $g \ln E, \mathrm{Ap}^{\mathrm{r}}$ & Jonsson et al. (2007) \\
\hline pGEX-GlnD & pGEX6P-3 derivative containing $g \ln D, \mathrm{Ap}^{\mathrm{r}}$ & Jonsson \& Nordlund (2007) \\
\hline pMJET & pET15b derivative containing $g \ln B, \mathrm{Ap}^{\mathrm{r}}$ & Johansson \& Nordlund (1999) \\
\hline pETGlnK & pET15b derivative containing $g \ln K, \mathrm{Ap}^{\mathrm{r}}$ & Jonsson et al. (2007) \\
\hline pETGlnJ & pET15b derivative containing $g \ln J, \mathrm{Ap}^{\mathrm{r}}$ & Jonsson et al. (2007) \\
\hline pETGlnJQ39E & pETGlnJ derivative encoding recombinant GlnJQ39E, $\mathrm{Ap}^{\mathrm{r}}$ & Jonsson \& Nordlund (2007) \\
\hline pETGlnJR47A & pETGlnJ derivative encoding recombinant GlnJR47A, $\mathrm{Ap}^{\mathrm{r}}$ & This study \\
\hline pETGlnJK58A & pETGlnJ derivative encoding recombinant GlnJK58A, Ap ${ }^{r}$ & This study \\
\hline
\end{tabular}

named pETGlnJR47A and pETGlnJK58A. All constructs were verified by sequencing.

Purification of R. rubrum GInB, GInJ, GInK and GInJ variants. All constructs used were pET15b derivatives, yielding proteins with an amino-terminal poly-histidine tag. GlnB, GlnK, GlnJ and all variants used (Q39E, R47A, K58A) were purified using HiTrap HP $1 \mathrm{ml}$ columns (GE Healthcare), according to a procedure described previously (Jonsson et al., 2007).

Purification of $\boldsymbol{R}$. rubrum GS, GInE and GInD. GlnD was purified as a glutathione $S$-transferase fusion protein and the tag was removed by on-column cleavage using Precission Protease (GE Healthcare), as described previously (Jonsson \& Nordlund, 2007). GS was purified from diazotrophically grown $R$. rubrum $\mathrm{S} 1$ and $\mathrm{GlnE}$ as a recombinant protein with a carboxy-terminal poly-histidine tag. The purification of GS and GlnE has been described previously (Jonsson et al., 2007).

Isolation of an $R$. rubrum membrane fraction and complex dissociation/reassociation assays. $R$. rubrum cultures were grown diazotrophically to $\mathrm{OD}_{600} 1.5$ (measured with an Ultrospec 1100 pro spectrophotometer; Amersham Biosciences) and then incubated with $20 \mathrm{mM} \mathrm{NH}_{4} \mathrm{Cl}$ for $30 \mathrm{~min}$, prior to harvesting by centrifugation at $3000 \mathrm{~g}$ for $15 \mathrm{~min}$. Whole cells were frozen as pellets in liquid nitrogen. Each pellet was resuspended in $10 \mathrm{ml} 100 \mathrm{mM}$ Tris/HCl, pH 7.4, supplemented with $1 \mathrm{mM}$ PMSF, $20 \mu \mathrm{g}$ DNase $\mathrm{I} \mathrm{ml}^{-1}, 0.5 \mathrm{mg}$ lysozyme $\mathrm{ml}^{-1}$ and one tablet of complete EDTA-free protease inhibitors (Roche), and was lysed by passage through a French press (twice) at 18000 p.s.i. $\left(1.2 \times 10^{8} \mathrm{~Pa}\right)$ and $4{ }^{\circ} \mathrm{C}$. Unbroken cells were pelleted by centrifugation at $3000 \mathrm{~g}$ for $20 \mathrm{~min}$ at $4{ }^{\circ} \mathrm{C}$, and the resulting supernatant was centrifuged for $1 \mathrm{~h}$ at $150000 \mathrm{~g}$. The resulting membrane fraction was washed twice by resuspension in $25 \mathrm{mM}$ Tris/ $\mathrm{HCl}, \mathrm{pH} 7.4$, and centrifugation $(1 \mathrm{~h}$ at $150000 \mathrm{~g}$ ). For the dissociation experiments, the membrane fraction was resuspended in $25 \mathrm{mM}$ Tris/ $\mathrm{HCl}, \mathrm{pH} 7.4$, and $150 \mu \mathrm{l}$ membrane fraction [corresponding to $1 \mathrm{mg}$ total protein as determined by the Lowry method (Lowry et al., 1951)] was added to different combinations of effectors: ATP $\left(\mathrm{Na}_{2} \mathrm{ATP} .3 \mathrm{H}_{2} \mathrm{O}\right), \mathrm{ADP}\left(\mathrm{Na}_{2} \mathrm{ADP}\right)$, $\mathrm{MnCl}_{2}, \mathrm{MgCl}_{2}$ and 2-OG, as indicated, in a final volume of $200 \mu \mathrm{l}$. The suspension was incubated for $1 \mathrm{~h}$ at room temperature with agitation and subjected to centrifugation for $30 \mathrm{~min}$ at $150000 \mathrm{~g}$. A $100 \mu$ volume of the supernatant fraction was collected and the remainder discarded. The membrane fractions were then resuspended in $200 \mu 125 \mathrm{mM}$ Tris/HCl, pH 7.4, and both fractions were analysed by Western blotting.

In the reassociation experiments the isolated membrane fraction was washed (twice) by resuspension in $25 \mathrm{mM}$ Tris/ $\mathrm{HCl}, \mathrm{pH} 7.4,2 \mathrm{mM}$ ATP, $10 \mathrm{mM} \mathrm{MnCl}_{2}$ and $5 \mathrm{mM}$ 2-OG for $1 \mathrm{~h}$ at room temperature, and subsequently centrifuged at $150000 \mathrm{~g}$. The supernatant fraction (of the first wash) was collected and the low-molecular-mass components were removed by gel filtration using PD-10 columns (GE Healthcare). This fraction was named wtGlnJ and used in subsequent experiments. The resulting membrane pellet was again washed with $25 \mathrm{mM}$ Tris/ $\mathrm{HCl}, \mathrm{pH} 7.4$, to remove traces of the lowmolecular-mass components and finally resuspended in $25 \mathrm{mM}$ Tris/ $\mathrm{HCl} \mathrm{pH} \mathrm{7.4.} \mathrm{A} 150 \mu \mathrm{l}$ volume of the membrane suspension (corresponding to $1 \mathrm{mg}$ total protein) was incubated with $7 \mu \mathrm{g}$ protein (wtGlnJ, GlnJ, GlnJQ39E, GlnJR47A, GlnJK58A or GlnB) and different combinations of effectors (ADP, ATP, $\mathrm{MnCl}_{2}$ and 2-OG), as indicated. In the case of wtGlnJ the amount refers to the total amount of protein $(7 \mu \mathrm{g})$ in the wtGlnJ fraction. The suspensions were incubated overnight at $4{ }^{\circ} \mathrm{C}$ with agitation and subjected to centrifugation at $150000 \mathrm{~g}$ for $30 \mathrm{~min}$. A $100 \mu \mathrm{l}$ volume of the supernatant was collected and named the unbound fraction. The remaining supernatant was discarded. The resulting membrane fractions were resuspended in $200 \mu \mathrm{l} 25 \mathrm{mM}$ Tris/ $\mathrm{HCl}, \mathrm{pH} 7.4$,

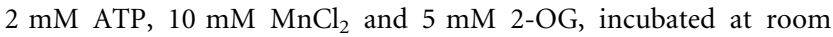
temperature for $1 \mathrm{~h}$, and centrifuged at $150000 \mathrm{~g}$ for $30 \mathrm{~min}$. The supernatant $(100 \mu \mathrm{l})$ was collected and named the rewash fraction. The remaining supernatant was discarded and the membranes were resuspended in $200 \mu \mathrm{l} 25 \mathrm{mM}$ Tris/HCl and saved.

All fractions were analysed using 12.5\% SDS-PAGE (Laemmli, 1970) followed by Western blotting using GlnJ antibodies. Western blots were developed using an LAS-1000 dark box (Fujifilm) and images were analysed with the MultiGauge v3.0 image analysis program (Fujifilm).

Uridylylation assays. The modification of PII proteins catalysed by GlnD was assessed by measuring the incorporation of $\left[\alpha_{-}{ }^{32} \mathrm{P}\right] \mathrm{UMP}$ after 20 min of incubation, according to Jonsson \& Nordlund (2007). Briefly, each reaction $(100 \mu \mathrm{l})$ contained $50 \mathrm{mM}$ HEPES, pH 7.6, $0.5 \mu \mathrm{M}$ PII protein (GlnJ, GlnJQ39E, GlnJR47A, GlnJK58A, GlnK or $\mathrm{G} \operatorname{lnB})$ or $1.5 \mu \mathrm{g}$ total protein when using the membrane wash fraction, $0.13 \mu \mathrm{M} \mathrm{GlnD}, 100 \mathrm{mM} \mathrm{KCl}, 1 \mathrm{mM}$ ATP, $1 \mathrm{mM}$ DTT, 
$0.5 \mathrm{mM}$ UTP $\left(\mathrm{Na}_{3} \mathrm{UTP}\right)$ supplemented with $0.5 \mu \mathrm{Ci}\left[\alpha^{32} \mathrm{P}\right] \mathrm{UTP}$ $\left(1.9 \times 10^{4} \mathrm{~Bq}\right), 0.3 \mathrm{mg} \mathrm{BSA} \mathrm{ml}^{-1}, 3 \mathrm{mM} \mathrm{MnCl}_{2}$, and $60 \mu \mathrm{M} 2-\mathrm{OG}$ or $25 \mathrm{mM} \mathrm{MgCl} 2$ and $250 \mu \mathrm{M} \mathrm{2-OG.} \mathrm{In} \mathrm{the} \mathrm{assays} \mathrm{in} \mathrm{which} \mathrm{the}$ ADP : ATP ratio was varied, ADP was at 1,2 or $5 \mathrm{mM}$ and $\mathrm{MnCl}_{2}$ was used (except in the assays with $\mathrm{GlnB}$, in which $\mathrm{MgCl}_{2}$ was used instead). Samples were withdrawn after $20 \mathrm{~min}$ incubation and mixed with loading buffer (130 mM Tris/HCl, pH 6.8, 4.2\% SDS, $20 \%$, v/v, glycerol, $10 \% 2$-mercaptoethanol and $0.003 \%$ bromophenol blue), boiled for 2 min and then run on $18 \%$ SDS-PAGE gels (Laemmli, 1970). After staining with Coomassie brilliant blue R250 the gels were dried and exposed for $12 \mathrm{~h}$ on a phosphoimager. Autoradiograms were analysed using the MultiGauge v3.0 program (Fujifilm).

Adenylylation assays. The effect of PII proteins and variants on adenylylation of GS catalysed by GlnE was assessed by measuring the remaining GS activity after $30 \mathrm{~min}$ of incubation, according to a procedure established previously (Jonsson et al., 2007). Briefly, each reaction $(100 \mu \mathrm{l})$ contained $50 \mathrm{mM}$ HEPES, $\mathrm{pH} 7.6,0.1 \mu \mathrm{M}$ GS, $0.1 \mu \mathrm{M}$ GlnE, $2 \mathrm{mM}$ ATP, $10 \mathrm{mM} \mathrm{MgCl}_{2}, 1.5 \mathrm{mg} \mathrm{BSA} \mathrm{ml}^{-1}, 0.5 \mu \mathrm{M}$ PII proteins (GlnB, GlnK, GlnJ, GlnJQ39E, GlnJR47A or GlnJK58A) and $1 \mathrm{mM}$ 2-OG, when indicated. In the assays in which the ADP : ATP ratio was varied, ADP was at 2, 4 or $10 \mathrm{mM}$. After $30 \mathrm{~min}$ incubation at $30{ }^{\circ} \mathrm{C}$ the GS activity was measured using the $\gamma$ glutamyl transferase reaction. Reaction mixtures without PII proteins were used as controls in each condition.

\section{RESULTS AND DISCUSSION}

\section{Initial considerations}

In the present study we chose to analyse the interaction of GlnJ with AmtB1 using wild-type $R$. rubrum instead of a $\Delta g \ln B g \ln K$ strain that was used in an earlier report (Wolfe et al., 2007). An ongoing study in our laboratory has shown that $a m t B 2$ is only weakly expressed compared with amtB1 (15-fold lower level) in wild-type $R$. rubrum under the experimental growth conditions used in this study $(\mathrm{H}$. Wang \& S. Nordlund, unpublished results). Based on this observation we assumed that the proportion of $A m t B 1$ in the membrane was much larger than that of AmtB2, and therefore that the latter would not interfere with our analysis. In addition, AmtB2 has never been shown to have any role in GlnJ sequestration to the membrane (Zhang et al., 2006b). These considerations, together with the recent observation that PII mutants in $R$. rubrum can develop suppressor mutations (Zhang et al., 2006a), for instance in $a m t B 1$, led us to perform this study in wild-type cells rather than in a PII mutant strain.

\section{Divalent cation dependence of the AmtB1-GInJ complex dissociation}

It has been shown recently that the in vitro uridylylation of PII proteins in $R$. rubrum by $G \ln D$ exhibits different divalent cation specificity for each PII protein, with GlnJ being uridylylated more extensively in the presence of $\mathrm{Mn}^{2+}$ compared with $\mathrm{Mg}^{2+}$, in a reaction requiring ATP and 2-OG (Jonsson \& Nordlund, 2007). With these results in mind, we investigated whether the release of GlnJ from R. rubrum membranes would show metal ion specificity, and also examined the dependence on ATP and 2-OG. The results depicted in Fig. 1(a) show that in the presence of $2 \mathrm{mM}$ ATP, $\mathrm{Mn}^{2+}$ promoted release of GlnJ more efficiently than $\mathrm{Mg}^{2+}$ at the same concentration, and for both ions the release was dependent upon the 2-OG concentration. Our results clearly differ from those observed for the Amt-GlnK complexes in Methanococcus jannaschii (Yildiz et al., 2007) and B. subtilis (Heinrich et al., 2006), in which 2-OG was not required for complex dissociation. However, the results are similar to those observed for the dissociation of the AmtB-GlnK complex in E. coli, for which maximum release requires 2-OG,

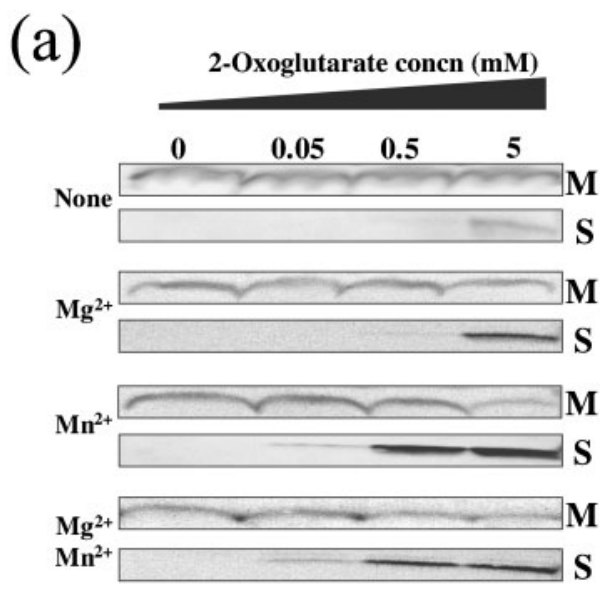

(b)
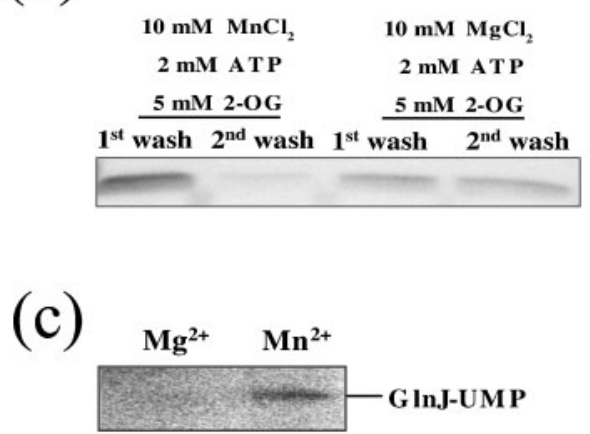

Fig. 1. Effect of divalent cations on wtGlnJ dissociation from membrane-bound AmtB1 and on uridylylation by $G \ln D$. Experiments were performed at least three times and results shown are from one representative experiment. (a) SDS-PAGE $(12.5 \%)$ followed by Western blotting using anti-GlnJ antibodies. R. rubrum membranes were washed with ATP $(2 \mathrm{mM}), \mathrm{MgCl}_{2}$ $(10 \mathrm{mM}), \mathrm{MnCl}_{2}(10 \mathrm{mM})$ and $2-\mathrm{OG}$ (as indicated), and the soluble (S) and membrane (M) fractions were analysed. (b) SDSPAGE (12.5\%) followed by Western blotting using anti-GinJ antibodies, showing the analysis (soluble fractions) of sequential washes with $2 \mathrm{mM}$ ATP, $5 \mathrm{mM}$ 2-OG and either $10 \mathrm{mM} \mathrm{MnCl}_{2}$ or $10 \mathrm{mM} \mathrm{MgCl} 2$. (c) Autoradiogram showing incorporation of $\left[\alpha^{-32} \mathrm{P}\right] \mathrm{UMP}$ in wtGlnJ by purified $R$. rubrum $\mathrm{GlnD}$ in the presence of ATP, 2-OG and either $\mathrm{MgCl}_{2}$ or $\mathrm{MnCl}_{2}$, as described in Methods. 
although extensive dissociation occurs in the presence of $\mathrm{MgCl}_{2}$ alone (Durand \& Merrick, 2006). Furthermore, the release of GlnJ was neither enhanced nor inhibited when both ions were present, compared with $\mathrm{Mn}^{2+}$ alone (Fig. 1a). The data in Fig. 1(a) clearly show that in the presence of $\mathrm{Mn}^{2+}$ not only is a higher amount of GlnJ released but also the release occurs at lower 2-OG concentrations when compared with the release in the presence of $\mathrm{Mg}^{2+}$. One possible explanation for this observation is that MnATP increases GlnJ affinity for 2-OG (when compared with MgATP); alternatively, the binding of $\mathrm{Mn}^{2+}$ or MnATP may in fact stabilize the T-loop in a conformation that favours complex dissociation.

In addition, Fig. 1(b) shows that the small amount of protein that remains at the membrane after incubation with ATP/ $\mathrm{Mn}^{2+} / 2-\mathrm{OG}$ is resistant to further washing steps. However, a second wash with $\mathrm{ATP} / \mathrm{Mg}^{2+} / 2-\mathrm{OG}$ results in additional recovery of GlnJ. Although there is no obvious explanation for this observation, it is clear that when $\mathrm{Mn}^{2+}$ is used, the GlnJ dissociation equilibrium is driven to a larger extent towards the soluble form while the same is not observed for $\mathrm{Mg}^{2+}$. As mentioned earlier in this section, it can be hypothesized that $\mathrm{Mn}^{2+}$ or MnATP provides greater stability to the soluble form, compared with $\mathrm{Mg}^{2+}$ or MgATP.

We also found that other divalent cations such as $\mathrm{Co}^{2+}$ and $\mathrm{Zn}^{2+}$ could produce an effect similar to that of $\mathrm{Mn}^{2+}$ on GlnJ release from the membrane, while $\mathrm{Ca}^{2+}$ or $\mathrm{Cu}^{2+}$ had an almost negligible effect (results not shown). Although these different effects can be understood from the different coordination chemistry of the cations studied, we believe that neither $\mathrm{Co}^{2+}$ nor $\mathrm{Zn}^{2+}$ has any physiological role in the regulation of GlnJ activity or association with AmtB1.

Taken together, our observations support an earlier report that shows the dependence on 2-OG and ATP concentrations, although in that study the effect of different metal ions was not analysed (Wolfe et al., 2007).

To analyse the relationship between GlnJ dissociation from the membrane and uridylylation, we dissociated GlnJ from R. rubrum membranes (with $\mathrm{ATP} / \mathrm{MnCl}_{2} / 2-\mathrm{OG}$ ) and then removed the effectors using gel filtration. We then analysed the uridylylation of the GlnJ present in this fraction (wtGlnJ) by purified $R$. rubrum $\mathrm{GlnD}$ in the presence of either $\mathrm{Mg}^{2+}$ or $\mathrm{Mn}^{2+}$, with ATP and 2-OG included (Fig. 1c). In fact, the wtGlnJ fraction was only uridylylated in the presence of $\mathrm{Mn}^{2+}$ (with ATP and 2-OG). It is thus clear that the same conditions are required for release of GlnJ as for efficient uridylylation catalysed by GlnD. This is also logical from a physiological point of view.

\section{The ADP/ATP ratio affects GInJ interaction with AmtB1, modification by GInD and stimulatory effect on GInE}

It has been shown recently in $R$. rubrum that the stability of the AmtB1-GlnJ complex is influenced by the ADP:ATP ratio. In fact, in the presence of ATP and 2-OG (two effectors for complex dissociation), the stability of the complex increases with increasing ADP : ATP ratio (Wolfe et al., 2007). In this study we also observed a decrease in the amount of GlnJ released from the membrane with an increase in the ADP: ATP ratio (Fig. 2a), in agreement with the earlier observation. Also, a recent in vitro study has clearly shown that the interaction of E. coli $\mathrm{GlnB}$ with the cellular targets $\mathrm{GlnE}, \mathrm{Gln} D$ and $\mathrm{NtrB}$ is affected by the ADP : ATP ratio (Jiang \& Ninfa, 2007). In this system, ADP competes with ATP for binding to $\mathrm{Gln} B$, and reduces the affinity of $\mathrm{GlnB}$ for 2-OG, thereby relieving the nitrogenlimitation signal. Considering these results we decided to extend our observations to other cellular targets by analysing the effect of changing the ADP:ATP ratio on the ability of GlnJ to stimulate GS adenylylation by GlnE and also on GlnJ uridylylation by GlnD. It is clear that in vitro GlnJ uridylylation (assessed as incorporation of $\left[\alpha-{ }^{32}\right.$ P]UMP) decreases with the increase in ADP:ATP ratio (Fig. 2b). The results presented in Fig. 2(b) are from an experiment in which the ATP concentration was kept constant and the ADP concentration was varied, thereby changing the ADP:ATP ratio. However, to rule out the possibility that this effect was due to the increase in the total concentration of adenine nucleotides, we performed a similar experiment in which the ADP:ATP ratio was varied but the total nucleotide concentration was kept constant, and the same results were obtained (results not shown). The effect of different ADP : ATP ratios on GlnJ stimulation of the GlnE adenylylation activity (in the presence or absence of 2-OG) was also analysed (Fig. 2c). GlnE adenylylating activity was assessed by measuring the remaining GS activity after $30 \mathrm{~min}$, as described in a previous report (Jonsson et al., 2007). In the absence of 2OG, only a slight decrease in adenylylation activity was observed at high ADP:ATP ratios. However, in the presence of 2-OG the adenylylation activity of $\mathrm{GlnE}$ increased with the increase in ADP:ATP ratio (Fig. 2c). This result clearly suggests that the increase in ADP concentration decreases the effect of 2-OG. This conclusion can be extended to the interaction with $A m t B 1$ and $G \ln D$, as it is clear that ADP has an inhibitory effect on both GlnJ dissociation from AmtB1 and uridylylation (processes that are affected by 2-OG). The same effects were observed when the other two R. rubrum PII proteins, GlnB and $\mathrm{GlnK}$, were used in the $\mathrm{GlnE}$ and $\mathrm{Gln} D$ assays (results not shown). As shown for the E. coli system (Jiang \& Ninfa, 2007), it is possible that ADP acts by reducing the affinity of PII proteins for 2-OG in R. rubrum also. Therefore, it seems that the regulatory features described for the nonnitrogen-fixing bacterium $E$. coli also apply in general terms to the photosynthetic nitrogen-fixing bacterium $R$. rubrum. In this sense our results are important because they show that the regulatory strategies in terms of energy signalling are similar between E. coli and R. rubrum, two bacteria with different physiological characteristics. It is becoming clear that the integration of energy signals by PII proteins occurs through the sensing of the cellular 

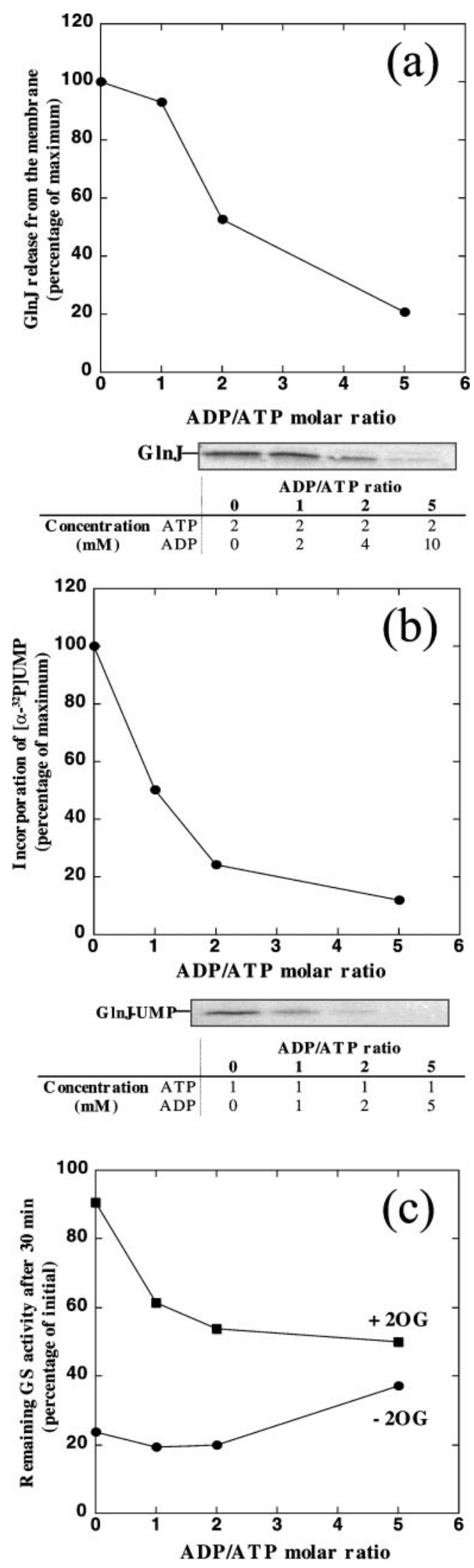

Fig. 2. Effect of different ADP/ATP ratios on GlnJ release from AmtB1, the adenylylation activity of $G \ln E$ and the uridylylation activity of $\mathrm{G} \operatorname{lnD}$. (a) Isolated $R$. rubrum membranes were washed with ATP (2 mM), $\mathrm{MnCl}_{2}$ (10 mM), 2-OG (500 $\left.\mu \mathrm{M}\right)$ and $\operatorname{ADP}(2,4$ and $10 \mathrm{mM})$. In each case the soluble fraction was analysed by SDS-PAGE followed by Western blotting with anti-GlnJ antibodies. The intensity of the bands in the Western blot was quantified and plotted as a function of ADP/ATP ratio. The results shown are from one representative experiment. (b) Incorporation of $\left[\alpha-{ }^{32} \mathrm{P}\right] \mathrm{UMP}$ into recombinant GlnJ by purified $R$. rubrum GlnD. ATP was at $1 \mathrm{mM}$ and ADP at 1,2 or $5 \mathrm{mM}$, as described in Methods. Both the autoradiogram and the corresponding quantification values are from one representative experiment. (c) Remaining GS activity after 30 min incubation with GlnE and GlnJ, in the presence or absence of $1 \mathrm{mM} 2-\mathrm{OG}$, with $2 \mathrm{mM}$ ATP and ADP at 2, 4 or $10 \mathrm{mM}$. GS activity was measured using the $\gamma$ glutamyl transferase reaction and the results are shown as percentages of initial activity. Results shown are the mean of three experiments.

ADP:ATP ratio, although more studies in different organisms are required to prove its universality.

In the case of R. rubrum, a number of studies have suggested that the regulation of nitrogen fixation and assimilation processes is intimately linked to cellular energy status (Zhang et al., 2001, 2006b). Different studies by several research groups have addressed this question by measuring the fluctuations of cellular adenine nucleotide pools (mainly ADP and ATP) upon addition of ammonium to nitrogenfixing cultures or after subjecting cells to darkness (Li et al., 1987; Nordlund \& Höglund, 1986; Paul \& Ludden, 1984). However, the lack of consistent data concerning the variation of ATP and ADP concentrations does not allow a firm conclusion to be drawn, as different growth conditions were employed. Nevertheless, the only assessment of nucleotide pools performed using $R$. rubrum cultures grown under $\mathrm{N}_{2}$ (conditions identical to those used in the present study) points to an increase in the ADP : ATP ratio from 0.2 to $0.5,3$ min after ammonium addition (Nordlund \& Höglund, 1986). It is interesting that a change in this range of concentrations would induce a strong response for $\mathrm{GlnE}$ and $\mathrm{GlnD}$, but not for the AmtB1GlnJ complex. As shown in Fig. 2(a) and by others (Wolfe et al., 2007), the effect on the AmtB1-GlnJ complex is only observed at ADP : ATP > 1 . However, even if the ADP : ATP ratios observed do not favour complex formation, this is known to occur upon ammonium addition (Zhang et al., 2006b). It is then more likely that AmtB1-GlnJ complex formation is controlled by the 2-OG concentration, and we have observed a decrease in the cellular 2-OG levels upon addition of ammonium to an $\mathrm{N}_{2}$-grown culture (P. F. Teixeira \& S. Nordlund, unpublished results).

\section{Reversible membrane association of GInJ is dependent on the concentration of 2-OG}

We also addressed the question of the reassociation of soluble GlnJ to the membrane-embedded AmtB1, using a 
different experimental setup (outlined in Fig. 3a) from the one used in Wolfe et al. (2007) to show the reversibility of GlnJ binding to AmtB1-containing membranes. In a first step, GlnJ was 'stripped' from the membrane using $10 \mathrm{mM}$ $\mathrm{MnCl}_{2}, 2 \mathrm{mM}$ ATP and $5 \mathrm{mM}$ 2-OG. Two fractions were obtained, the soluble one (containing GlnJ) and the pellet (with AmtB1-containing membranes). It was clear that most of GlnJ was in the soluble fraction at this point (insert in Fig. 3a) and that the fraction left at the membrane was resistant to further removal steps (Fig. 1b). Also, this

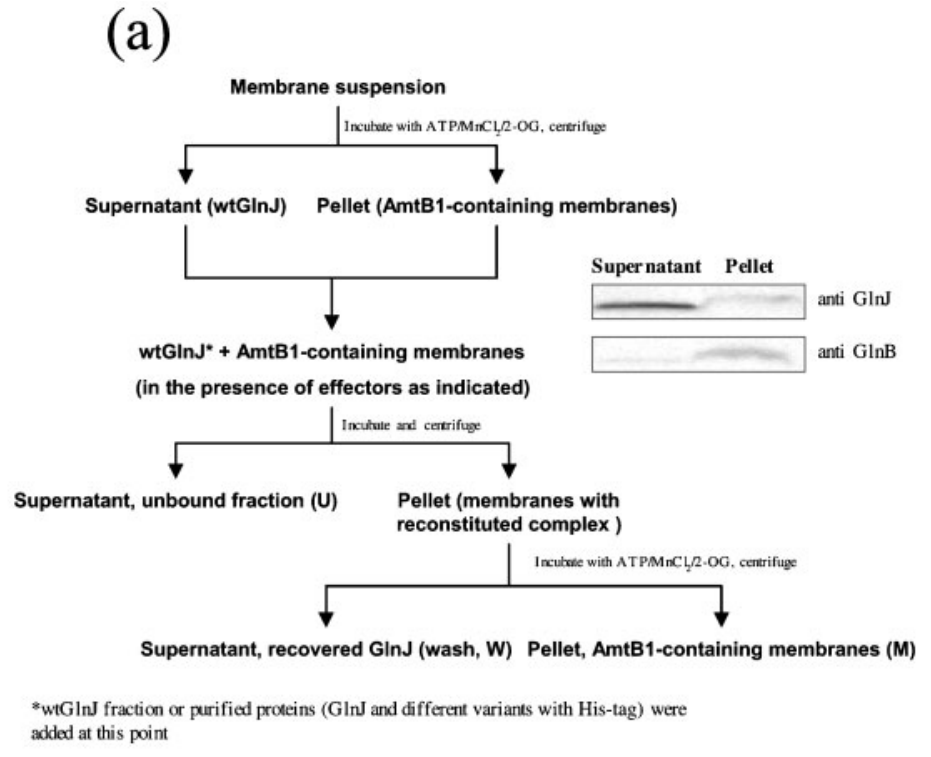

(b)
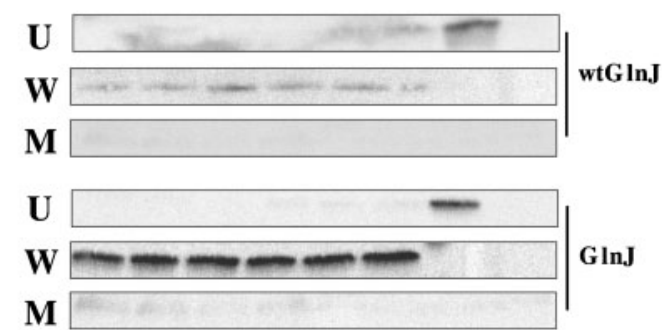

\begin{tabular}{|c|c|c|c|c|c|c|c|c|}
\hline Effector & 1 & 2 & 3 & 4 & 5 & 6 & 7 & 8 \\
\hline $\mathrm{ADP}(2 \mathrm{mM})$ & & $\mathrm{X}$ & & & & & & \\
\hline $\operatorname{ATP}(2 \mathrm{mM})$ & & & $\mathrm{X}$ & & $\mathrm{X}$ & $\mathrm{X}$ & $\mathrm{X}$ & \\
\hline $\mathrm{MnCl}_{2}(10 \mathrm{mM})$ & & & & $\mathrm{X}$ & $\mathrm{X}$ & $\mathrm{X}$ & $\mathrm{X}$ & \\
\hline 2-OG $(10 \mu \mathrm{M})$ & & & & & & $\mathrm{X}$ & & \\
\hline 2-OG $(1 \mathrm{mM})$ & & & & & & & $\mathrm{X}$ & \\
\hline Protein $(7 \mu \mathrm{g})$ & $X$ & $\mathrm{X}$ & $\mathrm{X}$ & $X$ & $\mathrm{X}$ & $\mathrm{X}$ & $\mathrm{X}$ & \\
\hline
\end{tabular}

(c)
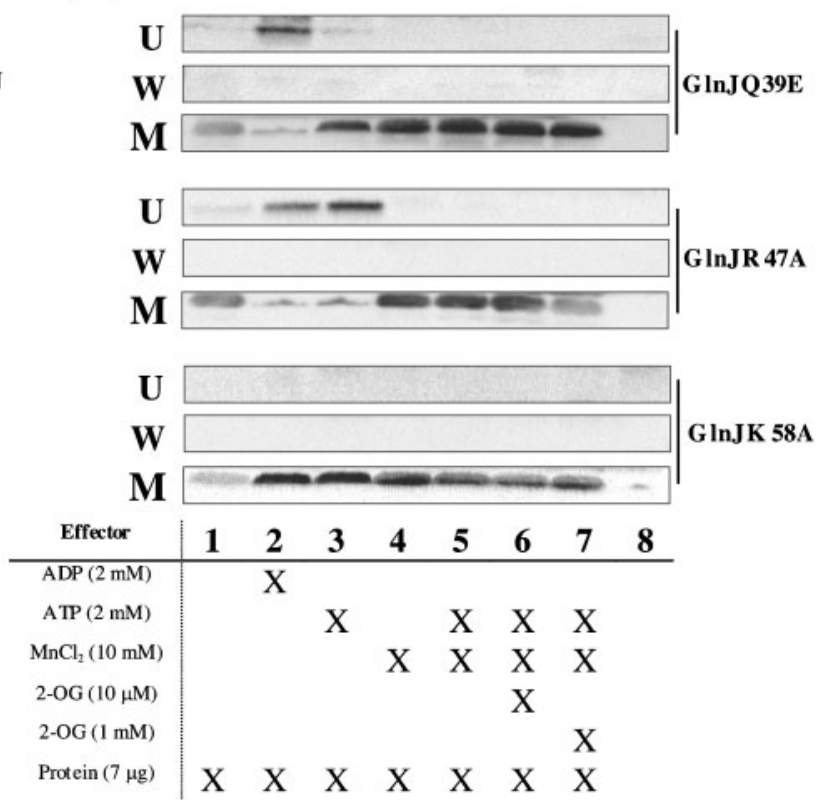

Fig. 3. Effectors influencing reversible GlnJ association with R. rubrum membranes. (a) Outline of the experimental setup. The samples analysed by Western blotting in each experiment are labelled as $\mathrm{U}$ (unbound), W (wash) and $\mathrm{M}$ (membrane) fractions. Insert shows Western blot analysis using either anti-GlnJ or anti-GlnB antibodies of both supernatant (wtGlnJ) and pellet (membrane) fractions after the first washing step. (b) AmtB1-containing membranes were incubated with wtGlnJ or purified GlnJ in the presence of effectors as indicated. After centrifugation the unbound fraction (U) was analysed by Western blotting. The reconstituted complexes at the membrane were dissociated with ATP/ $\mathrm{MnCl}_{2} / 2-\mathrm{OG}$ and the wash (W) and membrane (M) fractions were also analysed by Western blotting. Results shown are from one representative experiment. (c) A similar experimental setup to (b), but in this case the GlnJ variants Q39E, R47A and K58A were used. 
wtGlnJ fraction was essentially free of $G \ln B$, as shown (Fig. 3a) by the weak signal with $R$. rubrum $G \ln B$ antibodies, which in addition are known to react weakly with GlnJ. Although the GlnK levels cannot be quantified by Western blotting, since a specific antibody is not available, studies in our laboratory have shown that the expression of the $\operatorname{gln} K$ gene is 10 -fold lower than that of $\operatorname{gln} J$ (H. Wang \& S. Nordlund, unpublished results), and therefore it is unlikely that there is a substantial amount of $\mathrm{G} \operatorname{lnK}$ in this wash fraction. The low-molecular-mass components of the soluble fraction were then removed using gel filtration, and this fraction was named wtGlnJ. In a second step, we incubated GlnJ-stripped AmtB1-containing membranes with wtGlnJ in the presence of different effectors, and analysed the unbound (U) fraction (Fig. 3b). It should be noted that in each experiment the wtGlnJ fraction was later added to the same membrane fraction from which wtGlnJ had been obtained. In a third step, the bound wtGlnJ was again released by $10 \mathrm{mM} \mathrm{MnCl}_{2}, 2 \mathrm{mM}$ ATP and $5 \mathrm{mM}$ 2-OG, and both the soluble (W) and membrane (M) fractions were analysed (Fig. 3b). From these results we conclude that under these conditions the only factor that inhibits complex formation is the presence of 2-OG (Fig. 3b, fraction U). It is also important to note that the addition of metabolites was apparently not required to reconstitute the interaction.

Our results are similar to those reported for M. jannaschii, in which Amt1-GlnK1 complex formation occurs in vitro in the absence of any effector (Yildiz et al., 2007), unlike in E. coli (and A. brasilense), in which AmtB-GlnK (GlnZ) complex formation requires ATP (Durand \& Merrick, 2006; Huergo et al., 2007). The small differences in the intensity of the bands observed in the second wash (Fig. 3b, fraction W) likely arise from uneven resuspension of the membranes due to the small volumes used in each assay and thus do not affect the overall conclusion. The same experiment was repeated with the addition of purified GlnJ to AmtB1-containing membranes, and showed a similar result (Fig. 3b). Also, a similar pattern was obtained when using GlnB (results not shown), showing that GlnB has the putative structural requirements for binding to $A m t B 1$ and also that complex formation is inhibited by the same effector. The present results clearly support a role for 2-OG as the main effector controlling AmtB1-GlnJ complex formation (in the presence of ATP and $\mathrm{Mn}^{2+}$ ) and further reinforce our previous suggestion that $2-\mathrm{OG}$ is a fundamental signal controlling nitrogen assimilation in $R$. rubrum (Jonsson et al., 2007), which is also supported by the report of Wolfe et al. (2007).

\section{Interaction of GInJ T-loop variants with AmtB1}

PII signal transduction proteins exert their regulatory functions by direct protein-protein interactions with their cellular targets. Although the mechanism of interaction with the different targets is not completely understood, a number of studies have highlighted the importance of the
T-loop region for target interaction (Atkinson \& Ninfa, 1999; Conroy et al., 2007; Jiang et al., 1997; Pioszak et al., 2000).

Recently, it was reported that an $R$. rubrum GlnJQ39E variant is insensitive to the concentration of 2-OG, in an in vitro GlnE assay (Jonsson et al., 2007). Also, in E. coli, a GlnB Q39E variant exhibits impaired 2-OG binding and reduced ATP binding (Jiang et al., 1997).

With these results in mind we added the $R$. rubrum GlnJQ39E variant to AmtB1-containing membranes (after wtGlnJ had been removed) and analysed the unbound fraction. It was interesting to note that in this case it was possible to bind the protein to the membrane even when $1 \mathrm{mM}$ 2-OG was present (Fig. 3c). This observation confirms the previous proposal that this GlnJQ39E variant is insensitive to 2-OG. However, it was not possible to bind the protein in the presence of ADP, and also the binding was apparently reduced in the presence of ATP (in the absence of $\mathrm{Mn}^{2+}$ ). One possible reason for this observation is the excess negative charge due to nucleotide binding, exacerbating the effect of the Gln to Glu substitution. When $\mathrm{Mn}^{2+}$ is added together with ATP the negative charge is compensated by the metal ion and the binding ability is restored. Alternatively, in this variant the presence of $\mathrm{Mn}^{2+}$ might be required to stabilize the T-loop in the appropriate conformation to interact with $\mathrm{AmtB} 1$. We also tested whether the bound protein could be dissociated from AmtB1 using ATP and 2-OG. As shown in Fig. 3(c) (fractions $\mathrm{W}$ and $\mathrm{M}$ ), it was not possible to dissociate the bound protein. The GlnJQ39E variant bound to AmtB1 seems to be 'locked' in a conformation that is not sensitive to the metabolites added. Again, this experiment suggests that this variant is not affected by 2-OG and reinforces the role of this metabolite as the factor that controls the dynamics of the AmtB1-GlnJ complex.

A recent report on the structure of $M$. jannaschii $\mathrm{GlnK} 1$ has demonstrated for the first time the binding site for 2-OG and also that for MgATP (Yildiz et al., 2007). The structures determined show the difference between a form with an extended T-loop structure (the conformation that binds to Amt1) and a more compact one (that binds MgATP and 2-OG). It was proposed that the maintenance of the compact structure involved the formation of a salt bridge between Glu44 and Lys58, and that this compact conformation (with MgATP bound) creates the 2-OG binding site. Also, several reports have shown that the Arg47 residue is (in the extended conformation) on the tip of the T-loop and protrudes further into the AmtB vestibule (Conroy et al., 2007; Gruswitz et al., 2007). Based on the available structural information and due to the conservation of the aforementioned residues in $R$. rubrum GlnJ, we decided to analyse the importance of the Arg47 and Lys58 residues for complex formation with AmtB1 by constructing the GlnJ variants R47A and K58A. The R47A variant was able to associate to AmtB1containing membranes even in the presence of 2-OG 
(Fig. 3c). However, like the Q39E variant, it did not associate in the presence of ADP and ATP. The reason for this is probably the charge effect, as in the case of Q39E, as the addition of $\mathrm{Mn}^{2+}$ restores binding. However, complex dissociation was not observed in any variant. We can speculate that this difference represents a different type of binding to AmtB1, in a way that is not sensitive to the added metabolites.

In the case of the K58A variant (Fig. 3c) the association to AmtB1-containing membranes was possible under all conditions tested, but complex dissociation was not observed.

\section{Interaction of GInJ T-loop variants with the other cellular targets GInE and GInD}

After analysing the effect of the GlnJ Q39E, R47A and K58A substitutions on the interaction with AmtB1 we decided to investigate the effect of these variants with respect to two other cellular targets, GlnE and $G \ln D$. Interestingly, both Q39E and R47A variants were as effective as GlnJ in the activation of the adenylylating activity of GlnE, while the K58A variant showed a markedly decreased efficiency in this assay (Fig. 4a). We have

\section{(a)}

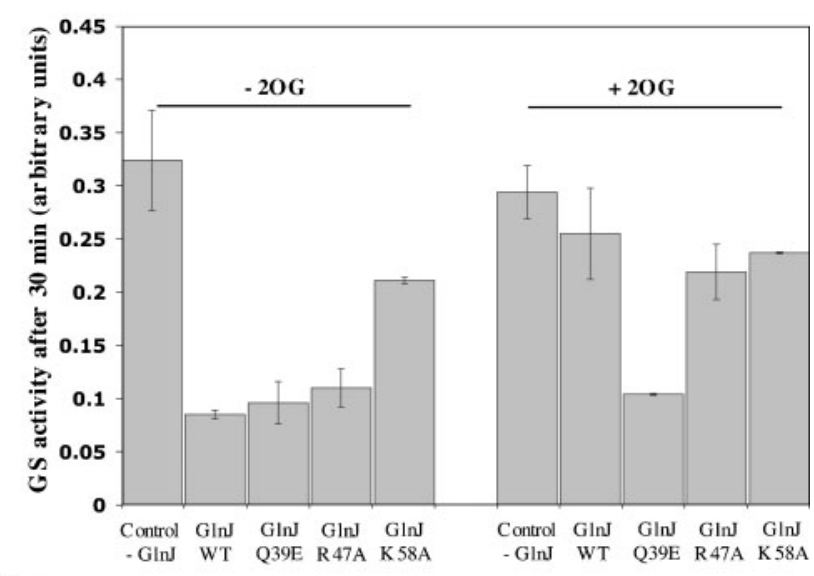

(b)

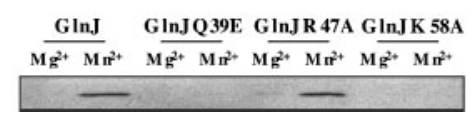

Fig. 4. Effect of GlnJ T-loop variants on GlnE activation and uridylylation by $\mathrm{GlnD}$. (a) Remaining GS activity after $30 \mathrm{~min}$ incubation with GlnE and GlnJ (or variants, as indicated) in the presence or absence of $1 \mathrm{mM} 2-\mathrm{OG}$. Results are from three independent experiments and are shown as mean $\pm \mathrm{SD}$. (b) Incorporation of $\left[\alpha^{-32} \mathrm{P}\right] \mathrm{UMP}$ into GlnJ (and variants, as indicated) by $R$. rubrum $\mathrm{GlnD}$ in the presence of ATP, 2-OG and either $\mathrm{MgCl}_{2}$ or $\mathrm{MnCl}_{2}$. Experiments were repeated at least three times and the results shown are from one representative experiment. previously reported that in the presence of 2-OG the PII proteins are unable to stimulate adenylylating activity of GlnE (resulting in no decrease in GS activity over time) and therefore we performed the same assay in the presence of $1 \mathrm{mM} 2-\mathrm{OG}$ to assess the sensitivity of the GlnJ variants to this effector in the adenylylation system. As reported previously (Jonsson et al., 2007), GlnJ is sensitive to 2-OG, resulting in inhibition of the GlnE adenylylation activity (i.e. no decrease in GS activity after $30 \mathrm{~min}$ ). Conversely, the Q39E variant was not sensitive to this metabolite, being equally efficient at stimulating GlnE even in the presence of 2-OG. The R47A variant was also, like GlnJ, sensitive to 2OG, while the K58A variant had a very similar effect with and without 2-OG, a low level of GlnE stimulation.

To get further insights into the interaction between the GlnJ variants and $\mathrm{GlnD}$, we tested whether the constructed variants could be modified by purified $R$. rubrum $\mathrm{GlnD}$ (in the presence of ATP, 2-OG and either $\mathrm{Mg}^{2+}$ or $\mathrm{Mn}^{2+}$ ). As expected, GlnJ was only modified in the presence of $\mathrm{Mn}^{2+}$, and a similar behaviour was observed for the R47A variant (Fig. 4b). In contrast, neither Q39E nor K58A could be modified under any of the conditions tested (Fig. 4b).

We have summarized the results of all our assays involving AmtB1, GlnE and GlnD in Table 2. Considering the results obtained with the three different variants it is possible to distinguish three distinct behaviours with respect to the interactions with the different targets. The R47A variant displayed characteristics similar to those of GlnJ, except in the interaction with AmtB1, in which it showed an unexpected behaviour. According to the structure of the E. coli AmtB-GlnK complex (Conroy et al., 2007), the R47 residue at the tip of the T-loop is fundamental for stopping ammonia conduction through the channel, constricting the exit of the cytoplasmic pore. This effect of GlnK is due to a network of hydrogen bonds and a salt bridge between the guanidinium group of R47 and two AmtB residues (S263 and D313). Because these residues are conserved in $R$. rubrum AmtB1 (data not shown), it is likely that similar interactions stabilize the AmtB1-GlnJ complex. Therefore, the underlying principle of the GlnJ R47A substitution is to disrupt this hydrogen bonding network with AmtB1, eventually leading to reduced binding of GlnJ to AmtB1. However, what we observed instead is that association of GlnJ to AmtB1 still occurs but is no longer inhibited by 2OG. The reason for this observation is not obvious, but could mean that in the AmtB1-GlnJR47A complex the binding mechanism is different and perturbs 2-OG binding to GlnJ. In spite of this apparent GlnJR47A resistance to 2OG binding when in complex with AmtB1, the metabolitesensing ability remains, as demonstrated by the GlnE and $\mathrm{GlnD}$ assays. In these two assays, the R47A behaviour is similar to that of GlnJ (including the response to 2-OG), indicating on one hand that the overall structure is not changed significantly by this substitution and on the other hand that the R47 residue does not play a significant role in the functional interactions with $\mathrm{GlnE}$ and $\mathrm{Gln} \mathrm{D}$ in vitro. 
Table 2. Summary of GlnJ interactions with different cellular targets

In each case, the processes observed are marked with + and the ones not observed with.$-(+)$ In this case the process was observed but with a decreased efficiency when compared with GlnJ.

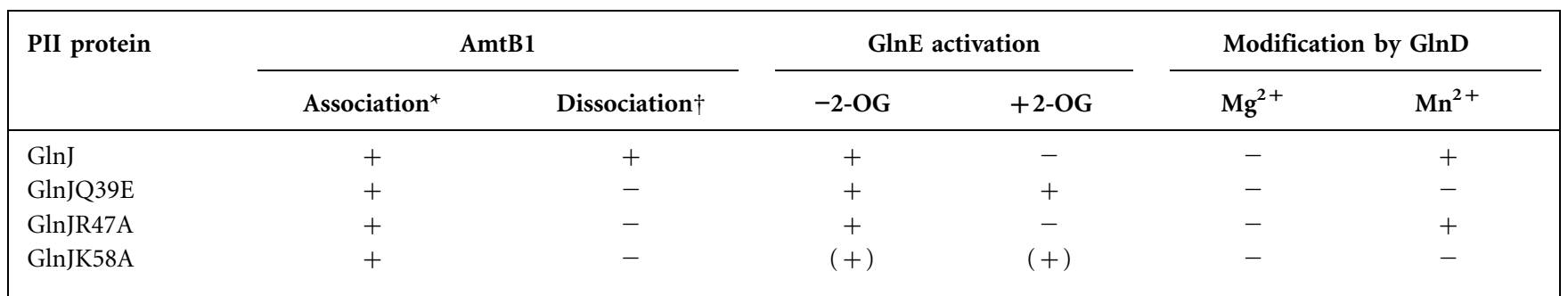

${ }^{*}$ Association in the absence of metabolites.

$\dagger$ Dissociation in the presence of ATP/ $\mathrm{Mn}^{2+} / 2$-OG.

The Q39E variant displayed a different pattern from that of R47A in the assays performed that was, however, consistent with the lack of 2-OG sensitivity. The effect of this substitution can be explained by considering the distinction between the extended and compact conformations of the T-loop in PII proteins that was recently observed for $M$. jannaschii GlnK1 (Yildiz et al., 2007); GlnK1 with bound MgATP and 2-OG presented a compact structure of the Tloop, while in the absence of 2-OG the T-loop was in an extended conformation. In the absence of metabolites the R. rubrum Q39E variant shows a pattern similar to that of GlnJ, being able to associate with AmtB1 and stimulate GlnE. However, this protein does not sense 2-OG, as seen by its inability to dissociate from AmtB1 (a process that requires 2-OG) and by its stimulation of $\mathrm{GlnE}$ in the presence of the same metabolite. It is clear that the structural requirements for target interaction involving the extended conformation are retained in this variant (as shown by the GlnE assay and by binding to AmtB1), but the compact conformation is probably not formed, resulting in an inability to dissociate from AmtB1 and to be uridylylated by GlnD. In the case of the $\mathrm{K} 58 \mathrm{~A}$ variant a third and different pattern of target interaction was distinguishable. This protein did associate with AmtB1, indicating that the structure of the T-loop in the extended conformation was kept, but did not dissociate (similar to the Q39E substitution), probably due to impaired transition to the compact form. In fact, for the formation of this compact form, the existence of a salt bridge between Lys58 and Glu44 is essential. A representation of the GlnJ 3D structure (model based on GlnK1 from M. jannaschii) and the interactions involving the Lys58 residue are shown in Supplementary Fig. S1. It is likely that the inability both to dissociate from AmtB1 and to be modified by GlnD are related to not being able to form a compact conformation (Supplementary Fig. S1a). However, the reduced stimulation of GlnE activity even in the absence of 2-OG (when compared with GlnJ and other variants) seems to indicate that this substitution induces a more global structural change in the protein, even in the extended conformation. The most obvious change is the inability to form a hydrogen bond with the carbonyl backbone of Gly87, a residue located in the B-loop (Supplementary Fig. S1). Therefore, it is likely that a K58A substitution affects at least the structure of the B-loop (and through this possibly metabolite binding), although other effects cannot be excluded. In this sense the results involving K58A are not as clear as those for Q39E and R47A, and their full understanding requires additional structural information.

In summary, we have highlighted here the importance of several T-loop residues for GlnJ interaction with different cellular targets, using specific variants designed on the basis of available structural data. We have shown that the Arg47 residue is important for the dynamics of AmtB1-GlnJ complex formation, while playing no major role in the interactions with either $\mathrm{G} \ln E$ or $\mathrm{G} \ln \mathrm{D}$. With respect to the Gln39 residue we further confirmed that this residue is required for target interaction involving the metabolite (ATP and 2-OG)-bound conformation. In a similar fashion, the Lys58 residue is necessary for interactions involving the compact conformation of the PII protein, although it is also fundamental to a more global maintenance of structure, as this residue is hydrogen bonded with the Gly87 residue in the neighbouring B-loop.

\section{Concluding remarks}

In the present report we further explore the regulatory properties of the multifunctional signal transduction PII proteins, highlighting factors that affect the interaction of GlnJ with cellular targets. Although the integration of nitrogen and carbon signals by PII proteins (by uridylylation and 2-OG binding, respectively) has been clearly established, the same is not true for the energy sensing, which was proposed only recently. The energy-sensing ability constitutes an additional feature with particular relevance in photosynthetic organisms. In this context it is interesting to compare the results we observed in $R$. rubrum with previously reported data for cyanobacteria with respect to the effect of the ADP:ATP ratio. In $R$. rubrum the ADP:ATP ratio affects PII modification (uridylylation), and also influences complex formation 
with AmtB1 and GlnE. It is known that PII dephosphorylation in cyanobacteria is not dependent on the ADP : ATP ratio (Forchhammer et al., 2004), but complex formation with $\mathrm{N}$-acetyl-L-glutamate kinase is affected by this signal, although in this case ADP induces complex dissociation (Maheswaran et al., 2004).

In conclusion, to understand the complexity and diversity of the regulatory strategies of PII proteins, further studies are required to determine, in addition to the bettercharacterized nitrogen and carbon signals, the relevance of energy sensing in both photosynthetic and non-photosynthetic bacteria and archaea.

\section{ACKNOWLEDGEMENTS}

The authors would like to thank Professor Gary Roberts, University of Wisconsin-Madison, for antibodies against R. rubrum GlnJ, and Dr Wally van Heeswijk, Free University, Amsterdam, for generously providing the E. coli strain RB9040. This study was supported by grants from the Swedish Research Council to S.N. and from Fundação para a Ciência e a Tecnologia (FCT), Portugal, through the PhD fellowship SFRH/BD/22162/2005 to P.F.T.

\section{REFERENCES}

Arcondeguy, T., Jack, R. \& Merrick, M. (2001). P(II) signal transduction proteins, pivotal players in microbial nitrogen control. Microbiol Mol Biol Rev 65, 80-105.

Atkinson, M. R. \& Ninfa, A. J. (1999). Characterization of the GlnK protein of Escherichia coli. Mol Microbiol 32, 301-313.

Bueno, R., Pahel, G. \& Magasanik, B. (1985). Role of $g \ln B$ and $g \ln D$ gene products in regulation of the glnALG operon of Escherichia coli. J Bacteriol 164, 816-822.

Conroy, M. J., Durand, A., Lupo, D., Li, X. D., Bullough, P. A., Winkler, F. K. \& Merrick, M. (2007). The crystal structure of the Escherichia coli AmtB-GlnK complex reveals how GlnK regulates the ammonia channel. Proc Natl Acad Sci U S A 104, 1213-1218.

Durand, A. \& Merrick, M. (2006). In vitro analysis of the Escherichia coli AmtB-GlnK complex reveals a stoichiometric interaction and sensitivity to ATP and 2-oxoglutarate. J Biol Chem 281, 29558-29567.

Forchhammer, K. \& Tandeau de Marsac, N. (1994). The PII protein in the cyanobacterium Synechococcus sp. strain PCC 7942 is modified by serine phosphorylation and signals the cellular $\mathrm{N}$-status. J Bacteriol 176, 84-91.

Forchhammer, K., Irmler, A., Kloft, N. \& Ruppert, U. (2004). PII signalling in unicellular cyanobacteria: analysis of redox-signals and energy charge. Physiol Plant 120, 51-56.

Gruswitz, F., O'Connell, J., III \& Stroud, R. M. (2007). Inhibitory complex of the transmembrane ammonia channel, AmtB, and the cytosolic regulatory protein, GlnK, at $1.96 \AA$. Proc Natl Acad Sci U S A 104, 42-47.

Heinrich, A., Woyda, K., Brauburger, K., Meiss, G., Detsch, C., Stulke, J. \& Forchhammer, K. (2006). Interaction of the membranebound GlnK-AmtB complex with the master regulator of nitrogen metabolism TnrA in Bacillus subtilis. J Biol Chem 281, 3490934917.

Hesketh, A., Fink, D., Gust, B., Rexer, H. U., Scheel, B., Chater, K., Wohlleben, W. \& Engels, A. (2002). The GlnD and GlnK homologues of Streptomyces coelicolor A3(2) are functionally dissimilar to their nitrogen regulatory system counterparts from enteric bacteria. $\mathrm{Mol}$ Microbiol 46, 319-330.

Huergo, L. F., Souza, E. M., Araujo, M. S., Pedrosa, F. O., Chubatsu, L. S., Steffens, M. B. \& Merrick, M. (2006). ADP-ribosylation of dinitrogenase reductase in Azospirillum brasilense is regulated by AmtB-dependent membrane sequestration of DraG. Mol Microbiol 59, 326-337.

Huergo, L. F., Merrick, M., Pedrosa, F. O., Chubatsu, L. S., Araujo, L. M. \& Souza, E. M. (2007). Ternary complex formation between $\mathrm{AmtB}, \mathrm{GlnZ}$ and the nitrogenase regulatory enzyme DraG reveals a novel facet of nitrogen regulation in bacteria. Mol Microbiol 66, 1523-1535.

Javelle, A., Severi, E., Thornton, J. \& Merrick, M. (2004). Ammonium sensing in Escherichia coli. Role of the ammonium transporter AmtB and AmtB-GlnK complex formation. J Biol Chem 279, 8530-8538.

Jiang, P. \& Ninfa, A. J. (2007). Escherichia coli PII signal transduction protein controlling nitrogen assimilation acts as a sensor of adenylate energy charge in vitro. Biochemistry 46, 12979-12996.

Jiang, P., Zucker, P., Atkinson, M. R., Kamberov, E. S., Tirasophon, W., Chandran, P., Schefke, B. R. \& Ninfa, A. J. (1997). Structure/function analysis of the PII signal transduction protein of Escherichia coli: genetic separation of interactions with protein receptors. J Bacteriol 179, 43424353.

Jiang, P., Peliska, J. A. \& Ninfa, A. J. (1998a). The regulation of Escherichia coli glutamine synthetase revisited: role of 2-ketoglutarate in the regulation of glutamine synthetase adenylylation state. Biochemistry 37, 12802-12810.

Jiang, P., Peliska, J. A. \& Ninfa, A. J. (1998b). Enzymological characterization of the signal-transducing uridylyltransferase/uridylylremoving enzyme (EC 2.7.7.59) of Escherichia coli and its interaction with the PII protein. Biochemistry 37, 12782-12794.

Johansson, M. \& Nordlund, S. (1996). Transcription of the $g \ln B$ and glnA genes in the photosynthetic bacterium Rhodospirillum rubrum. Microbiology 142, 1265-1272.

Johansson, M. \& Nordlund, S. (1999). Purification of P(II) and P(II)UMP and in vitro studies of regulation of glutamine synthetase in Rhodospirillum rubrum. J Bacteriol 181, 6524-6529.

Jonsson, A. \& Nordlund, S. (2007). In vitro studies of the uridylylation of the three PII protein paralogs from Rhodospirillum rubrum: the transferase activity of $R$. rubrum $\mathrm{GlnD}$ is regulated by $\alpha$ ketoglutarate and divalent cations but not by glutamine. J Bacteriol 189, 3471-3478.

Jonsson, A., Teixeira, P. F. \& Nordlund, S. (2007). The activity of adenylyltransferase in Rhodospirillum rubrum is only affected by $\alpha$ ketoglutarate and unmodified PII proteins, but not by glutamine, in vitro. FEBS J 274, 2449-2460.

Kamberov, E. S., Atkinson, M. R. \& Ninfa, A. J. (1995). The Escherichia coli PII signal transduction protein is activated upon binding 2ketoglutarate and ATP. J Biol Chem 270, 17797-17807.

Khademi, S., O'Connell, J., III, Remis, J., Robles-Colmenares, Y., Miercke, L. J. \& Stroud, R. M. (2004). Mechanism of ammonia transport by Amt/MEP/Rh: structure of AmtB at $1.35 \AA$. Science 305, 1587-1594.

Laemmli, U. K. (1970). Cleavage of structural proteins during the assembly of the head of bacteriophage T4. Nature 227, 680-685.

Li, J. D., Hu, C. Z. \& Yoch, D. C. (1987). Changes in amino acid and nucleotide pools of Rhodospirillum rubrum during switch-off of nitrogenase activity initiated by $\mathrm{NH}_{4}^{+}$or darkness. J Bacteriol 169, 231-237.

Lowry, O. H., Rosebrough, N. J., Farr, A. L. \& Randall, R. J. (1951). Protein measurement with the Folin phenol reagent. J Biol Chem 193, 265-275. 
Maheswaran, M., Urbanke, C. \& Forchhammer, K. (2004). Complex formation and catalytic activation by the PII signaling protein of $\mathrm{N}$ acetyl-L-glutamate kinase from Synechococcus elongatus strain PCC 7942. J Biol Chem 279, 55202-55210.

Merrick, M. J. \& Edwards, R. A. (1995). Nitrogen control in bacteria. Microbiol Rev 59, 604-622.

Nordlund, S. \& Höglund, L. (1986). Studies of the adenylate and pyridine nucleotide pools during nitrogenase "switch-off" in Rhodospirillum rubrum. Plant Soil 90, 203-209.

Nordlund, S. \& Ludden, P. W. (2004). Post-translational regulation of nitrogenase in photosynthetic bacteria. In Genetics and Regulation of Nitrogen Fixation in Free-Living Bacteria, pp. 175-196. Edited by W. Klipp, B. Masephol, J. R. Gallon \& W. E. Newton. Dordrecht, The Netherlands: Kluwer Academic Publishers.

Ormerod, J. G., Ormerod, K. S. \& Gest, H. (1961). Light-dependent utilization of organic compounds and photoproduction of molecular hydrogen by photosynthetic bacteria; relationships with nitrogen metabolism. Arch Biochem Biophys 94, 449-463.

Paul, T. D. \& Ludden, P. W. (1984). Adenine nucleotide levels in Rhodospirillum rubrum during switch-off of whole-cell nitrogenase activity. Biochem J 224, 961-969.

Pioszak, A. A., Jiang, P. \& Ninfa, A. J. (2000). The Escherichia coli PII signal transduction protein regulates the activities of the two-component system transmitter protein NRII by direct interaction with the kinase domain of the transmitter module. Biochemistry 39, 13450-13461.

Smith, C. S., Morrice, N. A. \& Moorhead, G. B. (2004). Lack of evidence for phosphorylation of Arabidopsis thaliana PII: implications for plastid carbon and nitrogen signaling. Biochim Biophys Acta 1699, 145-154.

Strosser, J., Ludke, A., Schaffer, S., Kramer, R. \& Burkovski, A. (2004). Regulation of GlnK activity: modification, membrane sequestration and proteolysis as regulatory principles in the network of nitrogen control in Corynebacterium glutamicum. Mol Microbiol 54, 132-147.

Thomas, G., Coutts, G. \& Merrick, M. (2000). The glnKamtB operon. A conserved gene pair in prokaryotes. Trends Genet 16, 11-14.
Wang, H., Franke, C. C., Nordlund, S. \& Noren, A. (2005). Reversible membrane association of dinitrogenase reductase activating glycohydrolase in the regulation of nitrogenase activity in Rhodospirillum rubrum; dependence on GlnJ and AmtB1. FEMS Microbiol Lett 253, 273-279.

Wolfe, D. M., Zhang, Y. \& Roberts, G. P. (2007). Specificity and regulation of interaction between the PII and AmtB1 proteins in Rhodospirillum rubrum. J Bacteriol 189, 6861-6869.

Xu, Y., Cheah, E., Carr, P. D., van Heeswijk, W. C., Westerhoff, H. V., Vasudevan, S. G. \& Ollis, D. L. (1998). GlnK, a PII-homologue: structure reveals ATP binding site and indicates how the T-loops may be involved in molecular recognition. J Mol Biol 282, 149-165.

Yildiz, O., Kalthoff, C., Raunser, S. \& Kuhlbrandt, W. (2007). Structure of GlnK1 with bound effectors indicates regulatory mechanism for ammonia uptake. EMBO J 26, 589-599.

Zhang, Y., Pohlmann, E. L., Ludden, P. W. \& Roberts, G. P. (2001). Functional characterization of three GlnB homologs in the photosynthetic bacterium Rhodospirillum rubrum: roles in sensing ammonium and energy status. J Bacteriol 183, 6159-6168.

Zhang, Y., Pohlmann, E. L. \& Roberts, G. P. (2005). GlnD is essential for NifA activation, NtrB/NtrC-regulated gene expression, and posttranslational regulation of nitrogenase activity in the photosynthetic, nitrogen-fixing bacterium Rhodospirillum rubrum. J Bacteriol 187, 1254-1265.

Zhang, Y., Pohlmann, E. L., Conrad, M. C. \& Roberts, G. P. (2006a). The poor growth of Rhodospirillum rubrum mutants lacking PII proteins is due to an excess of glutamine synthetase activity. Mol Microbiol 61, 497-510.

Zhang, Y., Wolfe, D. M., Pohlmann, E. L., Conrad, M. C. \& Roberts, G. P. (2006b). Effect of AmtB homologues on the post-translational regulation of nitrogenase activity in response to ammonium and energy signals in Rhodospirillum rubrum. Microbiology 152, 20752089.

Edited by: K. Forchhammer 